\title{
Mitotic memories of gene activity
}

\author{
Inma Gonzalez ${ }^{1,2}$, Amandine Molliex ${ }^{1,2}$ and Pablo Navarro ${ }^{1, *}$
}

1: Epigenomics, Proliferation and the Identity of Cells, Department of Developmental and Stem Cell Biology, Institut Pasteur, CNRS UMR3738, Paris, France.

2: Equal contribution

* Correspondence: pnavarro@pasteur.fr

\begin{abstract}
When cells enter mitosis, they undergo series of dramatic changes in their structure and function that severely hamper gene regulatory processes and gene transcription. This raises the question of how daughter cells efficiently recapitulate the gene expression profile of their mother such that cell identity can be preserved. Here, we review recent evidence supporting the view that distinct chromatin-associated mechanisms of gene regulatory inheritance assist daughter cells in the postmitotic reestablishment of gene activity with increased fidelity.
\end{abstract}




\section{Introduction}

Mitosis entails profound structural and functional cellular changes leading to major consequences for gene regulation [1]. On the one hand, the modifications to which chromatin is subject (e.g. hyperphosphorylation, condensation, topological rearrangements) drastically change the operational template of gene regulators such as Transcription Factors (TFs). On the other, the nuclear envelop breakdown leads to a sudden increase in the volume that gene regulators need to explore to maintain their chromatin-associated functions. Together, these changes converge on reduced gene regulatory capacity and a severe transcriptional downregulation. Hence, as daughter cells emerge and reconstitute a functional nucleus [2], numerous gene regulatory processes need to be restored to faithfully recapitulate the gene expression profile of the mother cell. However, cells dispose of mechanisms to "remember" gene regulatory states during mitosis, thereby reproducing gene activity more efficiently and accurately than if it would be de novo re-established after mitosis. While several mechanisms are certainly at play, we will focus here on two types involving chromatin-associated gene regulation. The first one can be considered as an active memory process, a short-term and fast mechanism whereby certain trans-regulators, particularly sequence-specific TFs, remain capable of interacting with the chromatin, either at specific regulatory elements and referred to as "mitotic bookmarking factors", or non-specifically [3]. The second one encompasses a variety of more stable chromatin characteristics, notably histone modifications and histone variants, which remain enriched at specific regulatory elements during mitosis, acting as "epigenetic marks" [4]. While the former can be associated with concepts such as working memory in neurosciences or live memory in computer sciences, the latter is analogical to long-term memory or storage, a concept that has been extensively studied for repressive mechanisms and only recently for gene activity, the focus of this review.

\section{Mitosis, a challenge for TF activity?}

During the phosphorylation cascade triggering entry into mitosis [5], several gene regulators are directly targeted. Among them, $\mathrm{C} 2 \mathrm{H} 2$ zinc-finger TFs (e.g. Sp1 and $\mathrm{Yy} 1$ ) are prevalent targets [6] but other TFs such as Oct4, a homeodomain TF of the POU family, or Sox2, an HMG-group TF, are also phosphorylated $[7,8]$. These phosphorylation events inhibit their sequence-specific DNA binding activity, leading to the eviction of these phosphorylated TFs from mitotic chromosomes. However, mitotic retention of TFs has been increasingly reported using proteomic strategies of mitotic chromosomes [9-11]. All these studies, highly variable in cell types and methodologies, show a clear enrichment for TFs, histone modifiers and chromatin remodelers. More specifically, TFs have been screened by live imaging for their capacity to remain associated to mitotic chromosomes [12], revealing a large number that are not fully evicted. These observations raise the possibility that mitosis may not represent a particular challenge for preserving gene regulatory processes. Adding to 
this notion, several maps of chromatin accessibility - often used as a proxy for TF activity - have now revealed that mitotic chromatin is not as condensed as previously thought, at least locally at promoters and enhancers [13-17]. However, careful examination of individual cases has led to richer and more complex scenarios, where it seems important to distinguish a global coating of the chromosomes by gene regulators from their proper site-specific interaction with regulatory elements [18]. For instance, several TFs have been shown to engage in site-specific interactions with the chromatin: the hematopoietic transcription factor GATA1 [19], the pioneer factor FoxA1 [20], the pluripotency TFs Esrrb [21] and KIf4 [22], as well as the architectural TF CTCF [23] and the general TF TBP [15]. Others, such as Sox 2 and Oct4, can globally interact in varying degrees with the chromosomes but not necessarily with their cognate DNA motifs [16].

\section{Mitotic bookmarking factors}

Despite the large differences in structure and biochemical properties among canonical mitotic bookmarking factors (Figure 1), several characteristics are shared by the aforementioned examples. For instance, during mitosis, these TFs bind some, although not all, of the targets they normally bind to during interphase. These sites tend to be enriched in DNA binding motifs of particularly high quality, indicating that mitotic bookmarking is primarily driven by sequence-specific interactions, as it was experimentally demonstrated for Esrrb [21]. Importantly, several correlations indicate that mitotic bookmarking represents a mechanism of gene expression memory preserving cell identity. Firstly, mitotic binding events are statistically enriched in the vicinity of genes rapidly reactivated after mitosis $[19,20,23,24]$; Secondly, genes responding to the activity of a given bookmarking TF early in $\mathrm{G} 1$ tend to be closer to bookmarked sites than genes responding at later cell cycle phases $[16,21]$. Thirdly, several bookmarking TFs are enriched in proximity of lineage-specific genes [19-21]. One key question is to understand how binding of these factors to specific targets during mitosis promote gene reactivation in daughter cells. Pioneering work indicated that mitotic bookmarking could be associated with preservation of chromatin accessibility [25]. Indeed, supporting this idea, TATA binding protein (TBP) specifically recruits the PP2A phosphatase at bookmarked promoters to inhibit the local action of condensins and maintain the chromatin accessible (Figure 2) [26]. However, mitotic depletion of TBP in pluripotent cells has not been associated with decreased accessibility [15], indicating that this mechanism may not be at play. More recently, it has been proposed that a mitotic specific complex centred on APC/C, is targeted to promoters in mitosis in order to trigger the local proteolysis of nucleosomes and to preserve or regain their accessibility (Figure 2) [27]. In fact, several lines of evidence suggest that nucleosome positioning and occupancy may be a parameter generally controlled by mitotic bookmarking factors. Indeed, both Esrrb and CTCF preserve very specific nucleosome architectures at their mitotic binding 
sites in pluripotent cells (Figure 1) [16,23]. In contrast, at sites losing binding of Esrrb, CTCF and other TFs such as Oct4 and Sox2, nucleosome positioning is lost. The maintenance of ordered nucleosomal arrays might facilitate the rapid reassembly of functional regulatory complexes to foster the reactivation of bookmarked genes after mitosis. In contrast, losing nucleosome order may occlude motifs for TFs (Figure 1), suggesting that daughter cells will require further chromatin remodelling processes to unmask them. Despite the recent progress in this field, clear experimental demonstrations of the importance of mitotic bookmarking are still lacking. In this regard, the example provided by Brd4, a ubiquitous coactivator involved in histone acetylation, is of primary importance. Initially considered to behave as a mitotic bookmarking factor [28], careful chemical inhibition of its activity during mitosis was later shown inconsequent regarding post-mitotic gene reactivation [29]. Whether all mitotic bookmarking factors display such a passenger or surrogate role to other activities - such as histone acetylation in the case of $\mathrm{Brd} 4$ - is an important possibility requiring further attention.

\section{Chromosome coating by TFs}

While the biochemical basis and the functional relevance of mitotic bookmarking factors are partially understood, how a large number of gene regulators accumulate at mitotic chromosomes, presumably non-specifically, and whether those exert any function remains elusive. Nevertheless, the invalidation at the mitosis-G1 transition of TFs coating mitotic chromosomes, such as Sox 2 and, to a lower extent Oct4, has detrimental physiological consequences, arguing for their functionality $[22,30]$. For instance, mitotic chromosomes could act as a reservoir of gene regulators in the close vicinity of DNA, thereby reducing the search time of their targets after mitosis (Figure 3 ). Also, these globally enriched factors could engage in specific interactions with their targets, but with kinetics fast enough to prevent current technologies capturing them. Such a mechanism of fast interactions by a multitude of different factors could be sufficient to preserve significant levels of chromatin accessibility during mitosis (Figure 3 ).

\section{Epigenetic chromatin states}

Active and repressed chromatin can be molecularly distinguished by several features such as combinations of post-translational modifications (PTMs) and the incorporation of distinct histone variants [31]. While these modifications are often instructed (directly or indirectly) by TFs or by other regulators (e.g. non-coding RNAs) to provide the required genomic specificity, they can be maintained even when their triggers are not any longer present. We consider this latter property as a defining characteristic of epigenetic regulation [32] of high relevance in the context of mitosis. Indeed, even when a gene regulator is mitotically inactivated, the changes it previously induced in 
the chromatin may persist during mitosis and convey gene regulatory information to the daughter cells. The study of these mitotically-associated histone marks can be traced back to more than three decades [33], primarily using immunofluorescence. More recently, proteomic analyses have underscored the prevalence of histone methylation in mitosis compared to acetylation, which is more largely reduced to facilitate chromatin compaction [34]. In this regard, we direct the reader to reviews that have more generally discussed chromatin states in mitosis [4] to focus more specifically on potential mechanisms of epigenetic inheritance of active transcription.

\section{Mitotic histone methylation at promoters}

As described for regions losing TF binding, promoters have also been shown to display dynamic organisations of the nucleosomes, whereby a deacetylated nucleosome invades the minimal promoter region spanning the transcription start site (Figure 2) [35]. Interestingly, it has been proposed that this may be due to the rolling backwards of the +1 nucleosome, containing the H2A.Z histone variant and preserving a mark associated with transcription, H3K4me3 (Figure 2) [36]. Hence, it has been suggested that this nucleosome relocation contributes to transcriptional silencing whilst priming genes for rapid reactivation via H3K4me3. However, these observations are not in agreement with other work performed in pluripotent cells, where it was observed that the nucleosome depleted regions of active promoters remain unoccupied by nucleosomes in mitosis [16]. Whether this is linked to the bookmarking capacity of TBP in these cells or to the proteasomal degradation of such nucleosomes via APC/C, remains unknow. Nevertheless, these data point to an important role of $\mathrm{H} 3 \mathrm{~K} 4 \mathrm{me} 3$ in preserving transcriptional potency through mitosis, as previously observed in hepatocytes [37] and in HeLa cells [38] . Similarly, H3K4me1, a generic mark of active regions, remains enriched at enhancers and insulators during mitosis (Figure 1) [39]. Therefore, in line with the relative stability of histone methylation, $\mathrm{H} 3 \mathrm{~K} 4 \mathrm{me} 1$ and $\mathrm{H} 3 \mathrm{~K} 4 \mathrm{me} 3$ display properties of epigenetic marks of active transcription during mitosis.

\section{Mitotic histone acetylation at enhancers and promoters}

Acetylation of $\mathrm{H} 3 \mathrm{~K} 27$ has emerged as a major mark of active regulatory elements, such as promoters and, even more prominently, enhancers. Unlike the global loss of histone acetylation, the mitotic behaviour of H3K27ac has been shown to be region and cell-type dependent. For instance, in immortalised human cell lines, H3K27ac is largely reduced during prometaphase, although some levels of H3K27ac remain at a subset of enhancers of cell type-specific genes with fast post-mitotic reactivation dynamics [39] (Figure 1). In mouse erythroblastic cells, however, the preservation of H3K27ac is more prominent, particularly at genes that are rapidly reactivated in early G1 [40]. This observation was later validated in pluripotent cells [22], where it was further shown that mitotic 
H3K27ac also characterises regions that rapidly reacquire the 3D topological structures associated with enhancer-promoter communications [24]. Although a limited number of cell types have been studied so far, these observations suggest a biological distinction between cancer and differentiated cells, which exhibit a relatively uniformly change in H3K27ac, and cells with developmental potency where changes in H3K27ac levels in mitosis are heterogeneous. More recently, a comprehensive study of histone acetylation (H3K14ac, H3K18ac, H4K5ac, H4K8ac, H4K12ac, H3K122ac, and H4K16ac) in mitotic erythroblastic cells was conducted, showing that a large number of acetyl-marks are preserved in mitotic chromatin and correlate with gene reactivation [29]. The mitotic preservation of H4K5ac at promoter regions was also observed in a human cancer cell line [41], but whether the cellspecific observation made for H3K27ac generally applies to all histone acetylation marks remains an interesting unknown possibility. Moreover, it has been postulated that HAT and HDAC enzymes are globally displaced from mitotic chromosomes [42] for proper chromosome condensation and mitotic progression [34]. How the equilibrium between acetylation/deacetylation is controlled at regulatory elements requires specific attention.

\section{Conclusions: current limitations and potential promises}

Through this review we have underscored three main mechanisms potentially facilitating gene reactivation in daughter cells (Figures 1 to 3 ), based either on non-specific or site-specific TF activity or on the preservation of chromatin properties. However, we still lack direct demonstrations of their functionality. This is a complicated task, since one would need to test the consequences of a lack of activity during mitosis exclusively, with an immediate restoration as the cells re-enter into interphase, entailing obvious experimental difficulties. Conceptual obstacles are also noticeable and will need to be solved as this field grows and tests its foundational principles (Figure 4). For instance, it is unclear if the consequences of a mitotic event should be sought early in the following interphase or, perhaps, in successive ones as the effects in gene regulatory networks could be strongly amplified or, on the contrary, buffered out [43]. Also, one should keep in mind that not all gene regulators are equal and some appear to be more powerful than others. For instance, CTCF regains full activity after mitosis very rapidly, regardless of its mitotic behaviour [44]. Moreover, it is today unclear how the distinct molecular strategies herein described influence each other, how they are orchestrated and whether they may display mutual compensation. Finally, it has recently been proposed that transcription around mitosis may be more complex than what we had anticipated. Firstly, mitosis itself may not be fully refractory to transcription; very low levels could persist and act as the most basic mechanism of memory $[45,46]$. Secondly, the genome seems to awake after mitosis as a strong transcriptional spike [40], a first burst of intense transcription that is then rechannelled in either sustained expression or repression as the enhancer and topological landscapes are rebuilt (Figure 4). 
In this context, the consequences of differential mitotic regulation may either be too subtle or, on the contrary, sufficiently robust to convey regulatory information over the initial transcriptional burst, subsequently contributing to the correct re-establishment of gene expression profiles.

\section{Figure legends}

Figure 1: Mitotic memory of gene regulation at enhancers. (A) During interphase, sequence-specific transcription factors (TFs) bind to their cognate DNA motifs within a nucleosome depleted region (NDR) flanked by ordered nucleosomes. These nucleosomes typically carry marks such as monomethylation of histone 3 at lysine 4 (H3K4me1, red circle) and acetylation of histone $\mathrm{H} 3$ at lysine 27 (H3K27ac, blue triangle). (B) During mitosis, TFs are evicted and nucleosomes invade the NDR, disorganising the nucleosomal array. This occludes DNA-binding motifs, further contributing to the loss of TF binding. Moreover, histone marks can also be erased. (C) Mitotic bookmarking TFs maintain an NDR and a nucleosome ordered array, which may prime the region for fast reactivation at mitosis exit (D) Histone modifications can also be mitotically maintained, marking the region for rapid post-mitotic re-activation.

Figure 2: Mitotic memory of gene regulation at promoters. (A) During interphase, the RNA Pol II preinitiation complex (PIC) is assembled at the Transcription Start Site (TSS), in the context of a nucleosome depleted region (NDR) with highly positioned +1 and -1 nucleosomes. These nucleosomes are characterised by marks such as acetylated H2A.Z and H3K4me3. (B) During mitosis, when the PIC disassembles, the +1 nucleosome rolls backwards into the NDR to occupy the TSS; several mechanisms have been described to maintain a memory of promoter activity. (C) The TSSassociated nucleosome may maintain $\mathrm{H} 2 \mathrm{~A} . \mathrm{Z}$ and $\mathrm{H} 4 \mathrm{~K} 4 \mathrm{me}$, promoting fast reactivation at mitotic exit. (D) Alternatively, APC/C may induce the degradation of this nucleosome to open the TSS and prime it for its reactivation in interphase. (E) The general TF TATA binding protein (TBP) may behave as a bookmarking factor.

Figure 3: Alternative mechanisms of mitotic memory. In addition to mitotic bookmarking and epigenetic marks, other mechanisms may contribute to a mitotic memory of gene regulation. (A) Multiple TFs may bind their specific DNA targets with very fast kinetics, collectively preserving chromatin accessibility. (B) TFs may be kept in the close vicinity of DNA without engaging in DNA- 
specific binding, acting like a reservoir. (C) Residual levels of transcription may be maintained during mitosis.

Figure 4: Mitotic bookmarking on the face of interphase dynamics. Top panel, mitosis:

Transcription is largely decreased during mitosis. Enhancer-promoter chromatin contacts are depleted during mitosis and restored rapidly upon G1 entry. The presence of mitotic bookmarking, chromosome coating and chromatin marks as well as the active but low transcription facilitate gene reactivation upon mitotic exit. The way these mechanisms influence each other and their relative importance are still unclear (squared-arrow network). Bottom panel, interphase: How the abovementioned mechanisms influence gene regulation before, during or after the transcriptional spike observed upon mitotic exit remains unknown, as does their temporal influence on how more complex regulatory networks are reestablished.

\section{Acknowledgements}

We thank our laboratory for critical discussions and Pedro Escoll for help with the illustrations. Work in P.N laboratory is supported by recurrent funding from the Institut Pasteur, the CNRS and Revive, as well as by funding obtained from the Agence Nationale de la Recherche (ANR 16 CE120004 01 MITMAT), the Ligue Nationale Contre le Cancer (LNCC EL2018 NAVARRO), and the European Research Council (ERC-CoG-2017 BIND).

\section{References}

1. Ma Y, Kanakousaki K, Buttitta L: How the cell cycle impacts chromatin architecture and influences cell fate. Front Genet 2015, 6.

2. de Castro IJ, Gokhan E, Vagnarelli P: Resetting a functional G1 nucleus after mitosis. Chromosoma 2016, 125:607-619.

3. Festuccia N, Gonzalez I, Navarro P: The Epigenetic Paradox of Pluripotent ES Cells. J Mol Biol 2017, 429:1476-1503.

4. Wang F, Higgins JMG: Histone modifications and mitosis: countermarks, landmarks, and bookmarks. Trends in Cell Biology 2013, 23:175-184.

5. Salaun P, Rannou Y, Claude P: Cdk1, Plks, Auroras, and Neks: The Mitotic Bodyguards. In Hormonal Carcinogenesis V. Edited by Li JJ, Li SA, Mohla S, Rochefort H, Maudelonde T. Springer; 2008:41-56. 
6. Rizkallah R, Alexander KE, Hurt MM: Global mitotic phosphorylation of $\mathbf{C 2} \mathbf{H} 2$ zinc finger protein linker peptides. Cell Cycle 2011, 10:3327-3336.

7. Shin J, Kim TW, Kim H, Kim HJ, Suh MY, Lee S, Lee H-T, Kwak S, Lee S-E, Lee J-H, et al.: Aurkb/PP1-mediated resetting of Oct4 during the cell cycle determines the identity of embryonic stem cells. elife 2016, 5:e10877.

8. Qi D, Wang Q, Yu M, Lan R, Li S, Lu F: Mitotic phosphorylation of SOX2 mediated by Aurora kinase $\mathbf{A}$ is critical for the stem-cell like cell maintenance in PA-1 cells. Cell Cycle 2016, 15:2009-2018.

9. Ohta S, Bukowski-Wills J-C, Sanchez-Pulido L, Alves F de L, Wood L, Chen ZA, Platani M, Fischer $L$, Hudson DF, Ponting CP, et al.: The protein composition of mitotic chromosomes determined using multiclassifier combinatorial proteomics. Cell 2010, 142:810-821.

10. Djeghloul D, Patel B, Kramer H, Dimond A, Whilding C, Brown K, Kohler A-C, Feytout A, Veland $\mathrm{N}$, Elliott J, et al.: Identifying proteins bound to native mitotic ESC chromosomes reveals chromatin repressors are important for compaction. Nature Communications 2020, 11:4118.

11. Ginno PA, Burger L, Seebacher J, lesmantavicius V, Schübeler D: Cell cycle-resolved chromatin proteomics reveals the extent of mitotic preservation of the genomic regulatory landscape. Nat Commun 2018, 9.

12. Raccaud M, Friman ET, Alber AB, Agarwal H, Deluz C, Kuhn T, Gebhardt JCM, Suter DM: Mitotic chromosome binding predicts transcription factor properties in interphase. Nature Communications 2019, 10:487.

13. Coux R-X, Owens NDL, Navarro P: Chromatin accessibility and transcription factor binding through the perspective of mitosis. Transcription 2020, 0:1-5.

14. Hsiung CC-S, Morrissey CS, Udugama M, Frank CL, Keller CA, Baek S, Giardine B, Crawford GE, Sung $\mathrm{M}-\mathrm{H}$, Hardison RC, et al.: Genome accessibility is widely preserved and locally modulated during mitosis. Genome Res 2015, 25:213-225.

15. Teves SS, An L, Bhargava-Shah A, Xie L, Darzacq X, Tjian R: A stable mode of bookmarking by TBP recruits RNA polymerase II to mitotic chromosomes. elife 2018, 7:e35621.

16. Festuccia N, Owens N, Papadopoulou T, Gonzalez I, Tachtsidi A, Vandoermel-Pournin S, Gallego E, Gutierrez N, Dubois A, Cohen-Tannoudji M, et al.: Transcription factor activity and nucleosome organization in mitosis. Genome Res 2019, 29:250-260.

17. Blythe SA, Wieschaus EF: Establishment and maintenance of heritable chromatin structure during early Drosophila embryogenesis. elife 2016, 5:e20148.

18. Festuccia N, Gonzalez I, Owens N, Navarro P: Mitotic bookmarking in development and stem cells. Development 2017, 144:3633-3645.

19. Kadauke S, Udugama MI, Pawlicki JM, Achtman JC, Jain DP, Cheng Y, Hardison RC, Blobel GA: Tissue-Specific Mitotic Bookmarking by Hematopoietic Transcription Factor GATA1. Cell 2012, 150:725-737. 
20. Caravaca JM, Donahue G, Becker JS, He X, Vinson C, Zaret KS: Bookmarking by specific and nonspecific binding of FoxA1 pioneer factor to mitotic chromosomes. Genes Dev 2013, 27:251-260.

21. Festuccia N, Dubois A, Vandormael-Pournin S, Gallego Tejeda E, Mouren A, Bessonnard S, Mueller F, Proux C, Cohen-Tannoudji M, Navarro P: Mitotic binding of Esrrb marks key regulatory regions of the pluripotency network. Nature Cell Biology 2016, 18:1139-1148.

22. Liu Y, Pelham-Webb B, Di Giammartino DC, Li J, Kim D, Kita K, Saiz N, Garg V, Doane A, Giannakakou P, et al.: Widespread Mitotic Bookmarking by Histone Marks and Transcription Factors in Pluripotent Stem Cells. Cell Reports 2017, 19:1283-1293.

23. Owens N, Papadopoulou T, Festuccia N, Tachtsidi A, Gonzalez I, Dubois A, Vandormael-Pournin $S$, Nora EP, Bruneau BG, Cohen-Tannoudji M, et al.: CTCF confers local nucleosome resiliency after DNA replication and during mitosis. eLife 2019, 8:e47898.

24. Pelham-Webb B, Polyzos A, Wojenski L, Kloetgen A, Li J, Giammartino DCD, Core L, Tsirigos A, Apostolou E: Mitotic retention of H3K27 acetylation promotes rapid topological and transcriptional resetting of stem cell-related genes and enhancers upon $\mathrm{G} 1$ entry. bioRxiv 2020, doi:10.1101/2020.06.02.130104.

25. Michelotti EF, Sanford S, Levens D: Marking of active genes on mitotic chromosomes. Nature 1997, 388:895-899.

26. Xing $\mathrm{H}$, Vanderford NL, Sarge KD: The TBP-PP2A mitotic complex bookmarks genes by preventing condensin action. Nat Cell Biol 2008, 10:1318-1323.

27. Oh E, Mark KG, Mocciaro A, Watson ER, Prabu JR, Cha DD, Kampmann M, Gamarra N, Zhou CY, Rape M: Gene expression and cell identity controlled by anaphase-promoting complex. Nature 2020, 579:136-140.

28. Dey A, Nishiyama A, Karpova T, McNally J, Ozato K: Brd4 Marks Select Genes on Mitotic Chromatin and Directs Postmitotic Transcription. Mol Biol Cell 2009, 20:4899-4909.

29. Behera V, Stonestrom AJ, Hamagami N, Hsiung CC, Keller CA, Giardine B, Sidoli S, Yuan Z-F, Bhanu NV, Werner MT, et al.: Interrogating Histone Acetylation and BRD4 as Mitotic Bookmarks of Transcription. Cell Reports 2019, 27:400-415.e5.

30. Deluz C, Friman ET, Strebinger D, Benke A, Raccaud M, Callegari A, Leleu M, Manley S, Suter DM: A role for mitotic bookmarking of SOX2 in pluripotency and differentiation. Genes Dev 2016, 30:2538-2550.

31. Kouzarides T: Chromatin modifications and their function. Cell 2007, 128:693-705.

32. Berger SL, Kouzarides $\mathrm{T}$, Shiekhattar R, Shilatifard A: An operational definition of epigenetics. Genes Dev 2009, 23:781-783.

33. Turner BM: Acetylation and deacetylation of histone $\mathbf{H} 4$ continue through metaphase with depletion of more-acetylated isoforms and altered site usage. Experimental Cell Research 1989, 182:206-214. 
34. Cimini D, Mattiuzzo M, Torosantucci L, Degrassi F: Histone Hyperacetylation in Mitosis Prevents Sister Chromatid Separation and Produces Chromosome Segregation Defects. MBOC 2003, 14:3821-3833.

35. Javasky E, Shamir I, Gandhi S, Egri S, Sandler O, Rothbart SB, Kaplan N, Jaffe JD, Goren A, Simon I: Study of mitotic chromatin supports a model of bookmarking by histone modifications and reveals nucleosome deposition patterns. Genome Res 2018, 28:1455-1466.

36. Kelly TK, Miranda TB, Liang G, Berman BP, Lin JC, Tanay A, Jones PA: H2A.Z Maintenance During Mitosis Reveals Nucleosome Shifting on Mitotically Silenced Genes. Mol Cell 2010, 39:901-911.

37. Kouskouti A, Talianidis I: Histone modifications defining active genes persist after transcriptional and mitotic inactivation. EMBO J 2005, 24:347-357.

38. Blobel GA, Kadauke S, Wang E, Lau AW, Zuber J, Chou MM, Vakoc CR: A Reconfigured Pattern of MLL Occupancy within Mitotic Chromatin Promotes Rapid Transcriptional Reactivation Following Mitotic Exit. Molecular Cell 2009, 36:970-983.

39. Kang H, Shokhirev MN, Xu Z, Chandran S, Dixon JR, Hetzer MW: Dynamic regulation of histone modifications and long-range chromosomal interactions during postmitotic transcriptional reactivation. Genes Dev 2020, 34:913-930.

40. Hsiung CC-S, Bartman CR, Huang P, Ginart P, Stonestrom AJ, Keller CA, Face C, Jahn KS, Evans $P$, Sankaranarayanan $\mathrm{L}$, et al.: A hyperactive transcriptional state marks genome reactivation at the mitosis-G1 transition. Genes Dev 2016, 30:1423-1439.

41. Zhao R, Nakamura T, Fu Y, Lazar Z, Spector DL: Gene bookmarking accelerates the kinetics of post-mitotic transcriptional re-activation. Nat Cell Biol 2011, 13:1295-1304.

42. Kruhlak MJ, Hendzel MJ, Fischle W, Bertos NR, Hameed S, Yang X-J, Verdin E, Bazett-Jones DP: Regulation of Global Acetylation in Mitosis through Loss of Histone Acetyltransferases and Deacetylases from Chromatin. J Biol Chem 2001, 276:38307-38319.

43. Owens NDL, Gonzalez I, Artus J, Navarro P: Chapter 6 - Mitotic bookmarking by transcription factors and the preservation of pluripotency. In Stem Cell Epigenetics. Edited by Meshorer E, Testa G. Academic Press; 2020:131-153.

44. Zhang $\mathrm{H}$, Emerson DJ, Gilgenast TG, Titus KR, Lan $\mathrm{Y}$, Huang $\mathrm{P}$, Zhang $\mathrm{D}$, Wang $\mathrm{H}$, Keller CA, Giardine $B$, et al.: Chromatin structure dynamics during the mitosis-to-G1 phase transition. Nature 2019, 576:158-162.

45. Palozola KC, Donahue G, Liu H, Grant GR, Becker JS, Cote A, Yu H, Raj A, Zaret KS: Mitotic Transcription and Waves of Gene Reactivation During Mitotic Exit. Science (New York, NY) 2017, 358:119.

46. Liu Y, Chen S, Wang S, Soares F, Fischer M, Meng F, Du Z, Lin C, Meyer C, DeCaprio JA, et al.: Transcriptional landscape of the human cell cycle. PNAS 2017, 114:3473-3478.

Five annotated references $\&$ description 
Ref10: 1 point

Given the well-established problems posed by chemical crosslinking of mitotic chromosomes, this paper provides a native perspective of their composition, revealing an enrichment for gene regulators.

Ref12: 1 point

First imaging screen of the capacity of TFs to coat the mitotic chromosomes, underscoring a correlation of this property with their ability to explore chromatin.

Ref16: 2 points

This paper clarifies the distinction between TF coating and proper mitotic bookmarking, and provides the first correlation between mitotic binding and the preservation of nucleosome order.

Ref29: 2 points

Comprehensive analysis of histone acetylation across mitotic chromatin, which, associated to chemical inhibition of Brd4, suggest epigenetic marks may be dominant over mitotic bookmarking by gene regulators.

Ref44: 1 point

Correlative study of genome organisation and mitotic binding of CTCF, indicating that bookmarking provides a minor advantage for restructuring the genome. 
A
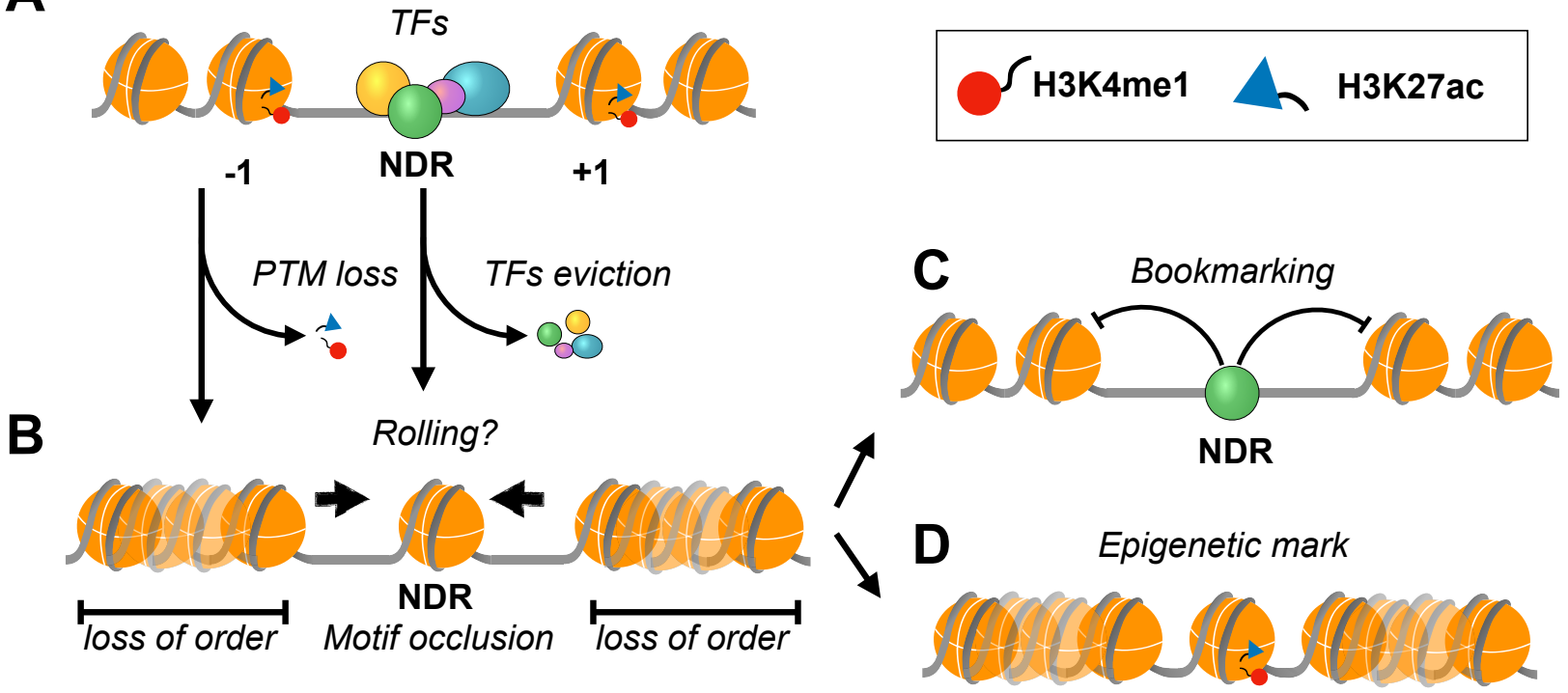

Figure 1 
A

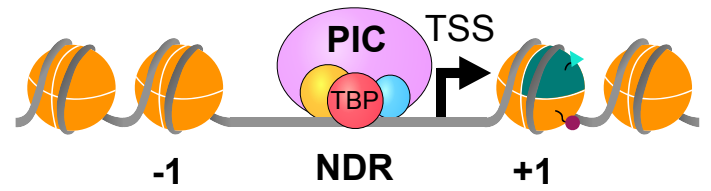

B

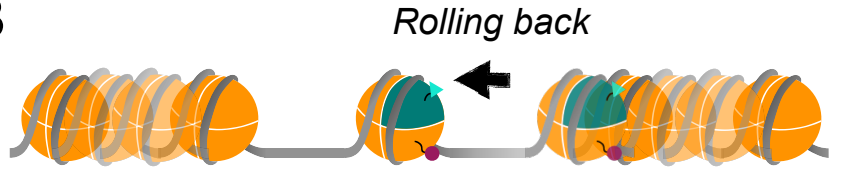

loss of order

H3K4me3
H2A.Z H2A.Z K2AC
C

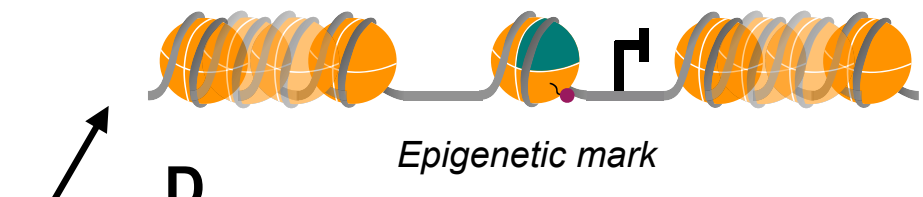

D

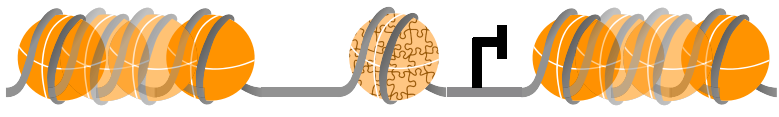

APC/C-dependent degradation

E

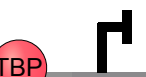

TBP bookmarking

Figure 2 


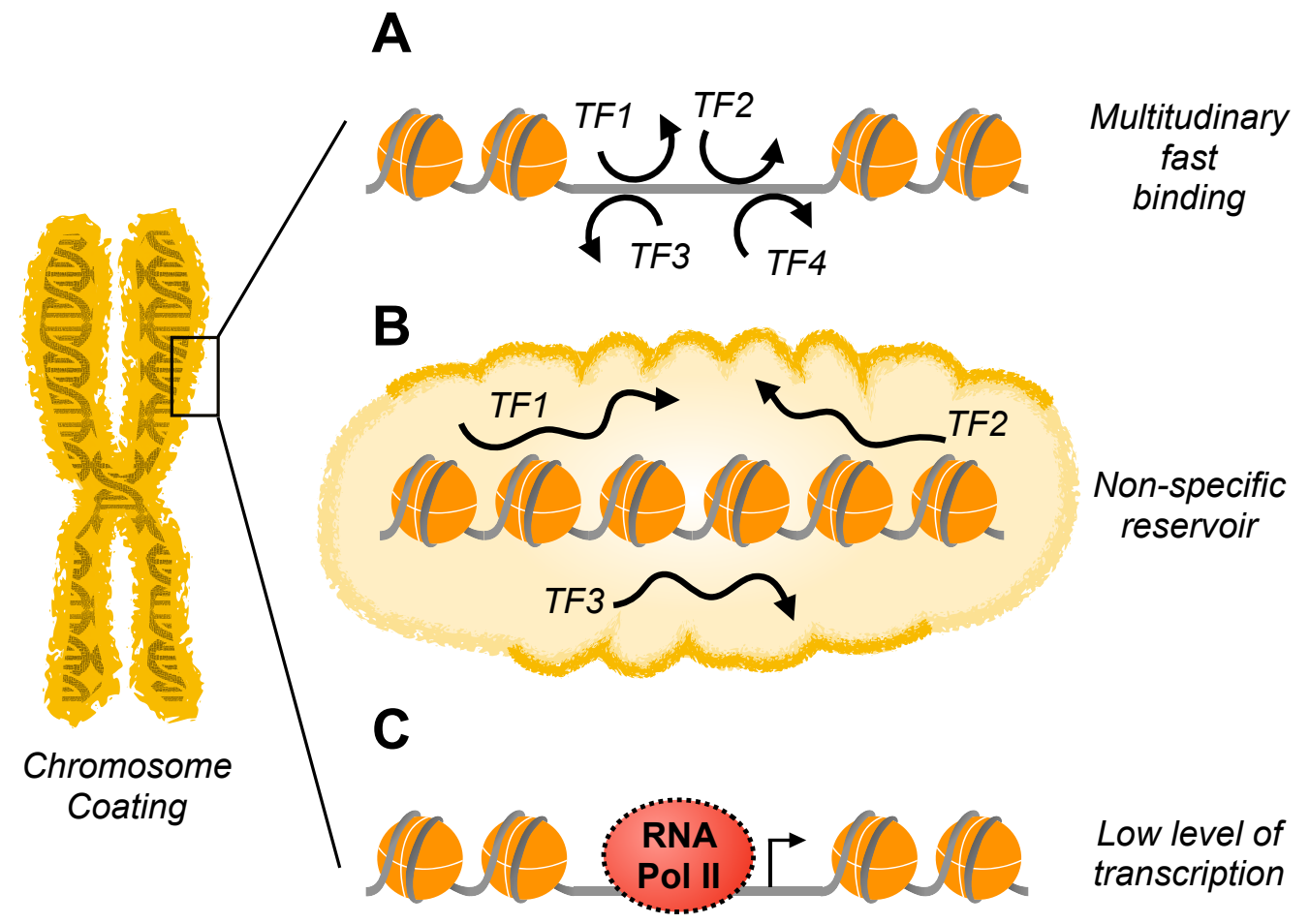

Figure 3 


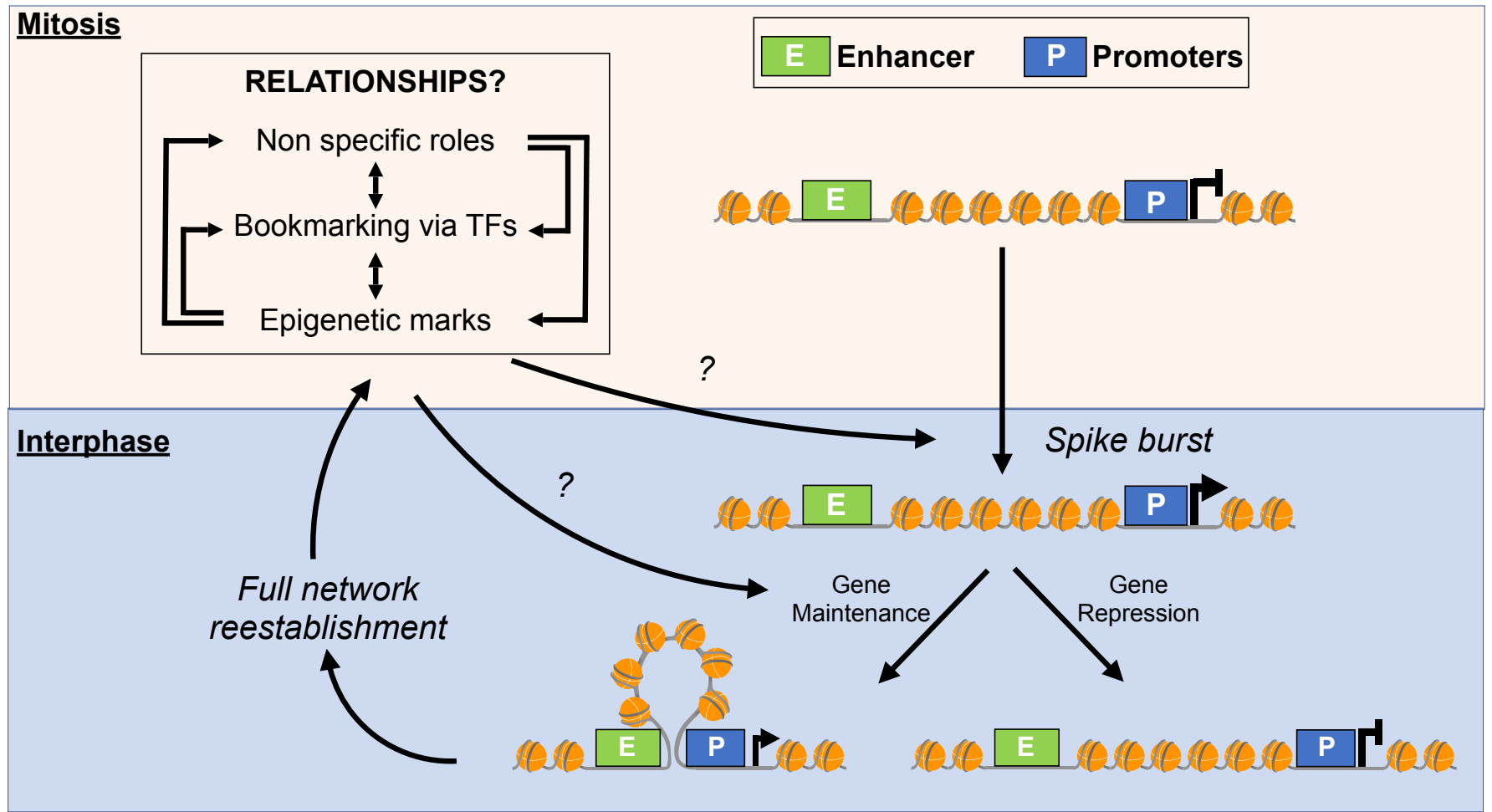

Figure 4 